\title{
Primary Frequency Regulation Comprehensive Analysis and Lean Evaluation of Generator Unit Based on Wide Area Measurement Information
}

\author{
Yuanhang Wang ${ }^{1}$, Dong $\mathrm{Liu}^{2, *}$, Guijun $\mathrm{Liu}^{1}$, Changwei Chen ${ }^{1}$, Bo Wang ${ }^{2}$ and Xin Shan ${ }^{2}$ \\ ${ }^{1}$ Grid Brazil Holding S.A., Av. Presidente Vargas, 955-SGCC Rio Tower, 15 andar, Centro, RJ, Rio de Janeiro, RJ, CEP: 20071- \\ 004 \\ ${ }^{2}$ NARI Group Corporation (State Grid Electric Power Research Institute), 19 Chengxin Avenue, Jiangning District, Nanjing, \\ Jiangsu Province, China \\ ${ }^{*}$ Corresponding author
}

\begin{abstract}
This paper presents a method that is primary frequency regulation(PFR) comprehensive analysis and evaluation based on wide area measurement information of generator unit, through analyzing the problems of the PFR test in the current PFR auxiliary service assessment. In this method, a combination of on-line monitoring and on-line testing is used to identify the PFR adjustment parameters of the generator unit. The PFR characteristic parameters and contribution effects of the generator unit are evaluated in different periods of frequency fluctuation based on the multi-dimensional refinement evaluation index. The effectiveness of the method is proved by application case analysis.
\end{abstract}

Keywords - generator units; primary frequency regulation(PFR) test; wide area measurement system (WAMS); comprehensive analysis, lean evaluation

\section{INTRODUCTION}

Primary frequency regulation (PFR) means that when grid frequency (cycle) deviates from the rated value, the regulation control system of generator in the grid will automatically control the increase (frequency drop) or decrease (frequency increase) of active power to limit the variation characteristics of grid frequency. It is an important link ${ }^{[1-2]}$ for regulation and control of both active power and frequency of a power system. It reflects the emergency response capacity of the power grid in the event of load. It plays an important role in the safe and stable operation of power system.

At present, the State Grid Corporation of China is in a transitional period of ultra-high voltage (UHV) grid construction, and AC/DC grid has a prominent chain effect. The grid was faced with severer safety situations. Only in 2015, there were several DC atresia accidents of grid happened. In "9.19" accident, the minimum grid frequency dropped to $49.563 \mathrm{~Hz}$, which seriously affected the safe and stable operation of grid. However, the mode of DEH+CCS is mostly adopted in the thermal power generator to realize PFR. The PFR circuit of many generators in DEH and CCS is subject to logic modification or switching operation through manual operation to make the generators unable to perform normally or totally lose the PFR. Therefore, it is of great significance in how to comprehensively analyze and leanly assess the actual generator PFR performance, to promote and improve the switch-in rate and usage rate of generator PFR, and to increase the operation quality and stability of grid.

In recent years, the gradual maturity and development ${ }^{[3]}$ of synchronized phase measuring technique based on global positioning system (GPS) technology work out the problem of synchronous data acquisition in different spatial positions, realize the synchronous acquisition of wide-area measured information of generator, and provide a new opportunity for on-line monitoring and analysis of generator PFR performance. Document [4] takes dead zone of frequency regulation and coefficient of differential adjustment as monitoring objects, Document [5] takes PFR contributing energy as the main performance assessment index, and Document [6] takes response speed, regulation range, offset and response index as the comprehensive assessment indexes. The above analysis methods are mostly based on generator output and characteristic curve of frequency variation to assess the generator PFR performance, which have engineering practicability. However, because the generator PFR is affected by operation conditions of generator and other factors, the response performance has larger dynamic characteristics. Meanwhile, simple on-line PFR monitoring method cannot conduct PFR performance analysis on shutdown generator during frequency disturbance either. Therefore, the PFR performance assessment index obtained cannot completely reflect the real effect of PFR. On the other hand, because phasor measurement unit (PMU) in wide area measurement system (WAMS) has regional distribution incompleteness, measured data finiteness and other problems, it is hard to realize complete and overall generator PFR performance analysis and assessment of the whole grid completely based on WAMS data. Therefore, some compensation measures have to be taken to process suspicious data, missing data of distribution points, etc. against the problem of analysis data limitation.

Therefore, based on WAMS and supervisory control and data acquisition (SCADA) hybrid data and wide-area measured information of generator, this article puts forward a comprehensive analysis and lean assessment method for PFR performance, establishes PFR assessment system combined with on-line PFR monitoring and testing, and applies to 
practical on-line generator PFR performance monitoring and assessment with good effect.

\section{PFR PERFORMANCE ASSESSMENT INDEX AND ANALYSIS METHOD PRINCIPLE}

\section{A. Comprehensive Performance Assessment Index}

The generator PFR performance is traditionally represented in droop of speed (differential coefficient) and slow rate (dead band) of steam turbine governing system. The two indexes are to reflect the inherent characteristics of generator unit. To reflect the regulation performance of generator unit, at present, the following indexes are mainly adopted in the "Implementation Rules for Management of Gird-connected Operation of Power Plant" in all the regions of China: PFR rate of putting into operation, PFR contributing energy, droop of speed, etc.

Nevertheless, in view of the actual stable operation of grid, speed ability and effectiveness shall be pursued in the initial period of PFR process to quickly regulate the frequency to the limited range. Unbiasedness shall be pursued in the later period to guarantee stable frequency variation ${ }^{[6]}$. This article synthesizes the performance requirements of PFR at different times and puts forward comprehensive analysis and assessment of generator PFR performance from regulating speed, regulation range, regulation precision, PFR comprehensive response and other dimensions as per output response index, piecewise energy contribution index, comprehensive index and multiple assessment indexes.

Main related performance indexes:

1) Regulating speed index $I_{s}[\%]$ : The time required from the grid frequency variation reaching to PFR operating value to the generator load starting to change toward to the direction of frequency recovery is called as response lag time. It reflects speed level of the generator PFR response, so the regulating speed index $I_{s}$ can be quantified through response lag time. Moreover, in the process of PFR assessment of Jiangsu Power Grid, to more fully reflect the speedability and effectiveness of generator PFR in the initial period, the regulating speed index $I_{s}$ was quantified through percentage of actual contributing energy accounting for theoretical contributing energy of generator PFR in the initial period.

$$
I_{s}=\frac{H}{H_{e}} \times 100 \%
$$

where, $H$ is the actual contributing energy of PFR in the initial period, and $H_{e}$ is the theoretical contributing energy of PFR in the initial period.

2) Regulation range index $I_{r}$ [\%]: After the grid frequency variation reaches PFR operating value, the generator load needs to be regulated precisely along with the frequency variation. In order to quickly regulate the frequency to the dead band, the generator load needs to be regulated quickly to a certain range. Therefore, the regulation range index $I_{r}$ can be quantified through percentage of actual maximum output regulating variable accounting for theoretical maximum output regulating variable of generator in the response time period of generator PFR.

$$
I_{r}=\frac{x_{1}}{y_{1}} \times 100 \%
$$

where, $x_{1}$ is the actual maximum output regulating variable of generator, and $y_{1}$ is the theoretical maximum output regulating variable of generator.

3) Regulation precision index $I_{c}[\%]$ : Within the generator PFR response time period starting from the frequency variation at the generator terminal exceeding the lower limit (or upper limit) of PFR dead band, the part that the active generated energy exceeds (or is short of) the energy generated in case that the frequency does not exceed the lower limit (or upper limit) of PFR dead band is called as PFR contributing energy. It reflects the contribution of the generator on load disturbance in the process of PFR. Therefore, the regulation precision index $I_{c}$ can be represented in the percentage of actual contributing energy accounting for theoretical contributing energy of generator PFR within the generator PFR response time period.

$$
I_{e}=\frac{P}{P_{e}} \times 100 \%
$$

where, $\mathrm{P}$ is the actual contributing energy of $\mathrm{PFR}$, and $P_{e}$ is the theoretical contributing energy of PFR.

4) Comprehensive response index $I_{e}[\%]$ : The comprehensive index comprehensively reflecting the generator PFR response performance obtained by calculating according to all components of regulating speed, regulation range and regulation precision as well as their weighting coefficients can be set based on the load characteristics of all the regional grids and the assessment criterion of "Implementation Rules for Management of Gird-connected Operation of Power Plant". Jiangsu Power Grid selected regulation range index and regulation precision index for comprehensive analysis and assessment.

$$
\begin{gathered}
I_{e}=(\text { weighting coefficient }) \times I_{r}+(\text { weighting coefficient }) \times I_{c} \\
+(\text { weighting coefficient }) \times I_{s}
\end{gathered}
$$

\section{B. Comprehensive Analysis and Lean Assessment Method Principle}

This article mainly centers on on-line PFR performance monitoring, supplemented by on-line testing. The combination 
of both is adopted to conduct comprehensive analysis and lean assessment to the generator PFR. On-line PFR performance monitoring reflects the actual generator PFR performance, and on-line PFR performance testing realizes on-line analysis of expost generator PFR performance and improves the comprehensiveness and precision of generator assessment. Both on-line monitoring and on-line testing supplement each other, which perfect PFR assessment function.

Firstly, the on-line generator PFR performance monitoring and testing functions acquire WAMS data and SCADA data on the current section in real time through smart grid dispatching technical supporting system, WAMS and SCADA hybrid data through time scale aligning, amendment, piecewise fitting and other data pre-processing methods, dynamically monitor the process of grid frequency variation, capture effective disturbance of the grid frequency, and recognize characteristic parameters and contributions of the generator PFR within different times of frequency fluctuation. Secondly, in combination of PFR characteristics of frequency fluctuation at different times, based on output response index, piecewise energy contribution index, comprehensive index and multiple assessment indexes, quantitatively assess the generator PFR performance within different times, comprehensively analyze and leanly assess the generator PFR performance, and establish generator PFR lean assessment system. The flowchart of comprehensive analysis and lean assessment method principle for generator PFR performance is as shown in Figure I.

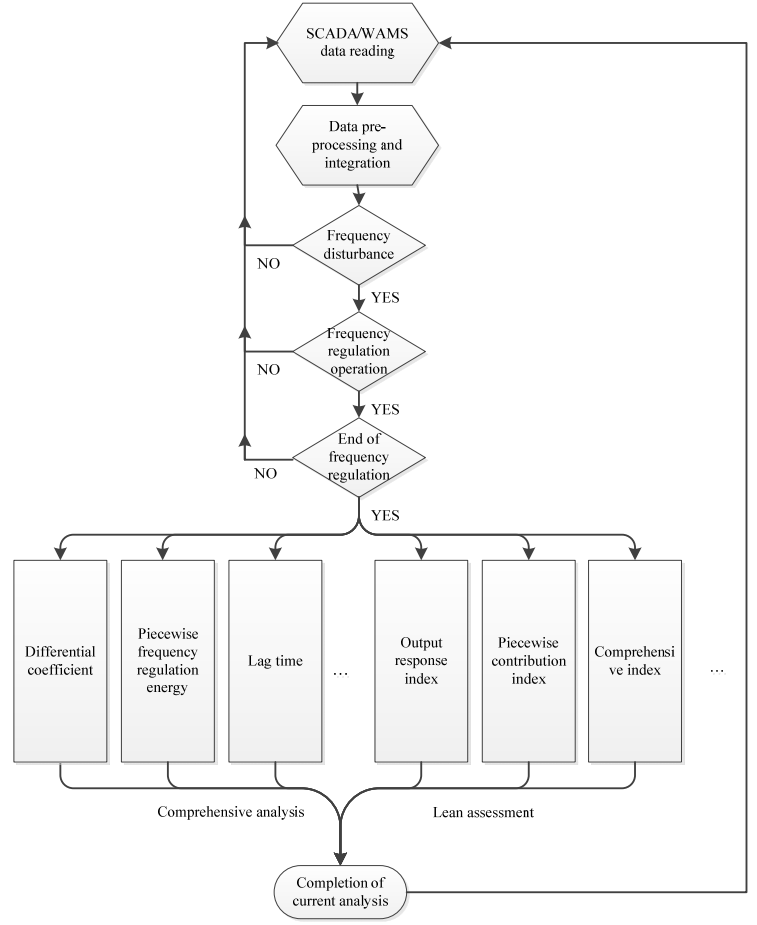

FIGURE I. FLOW CHART OF PFR METHOD PRINCIPLE

\section{WAMS AND SCADA DATA PRE-PROCESSING}

PMU based on GPS technology acquires voltage, current and other phasors as well as power, frequency and other analog quantities of the generator in the grid in real time, and sends to
WAMS master station ${ }^{[3]}$ in real time at a speed of 25 frames per second to 100 frames per second, but PMU may have some random disturbances and random failures in the process of data acquisition and data transmission, so there may have suspicious data and missing data in WAMS data. On the other hand, due to the incompleteness of regional distribution and the finiteness of measured data of PMU in WAMS, it may result in overall missing of data of some generators. The suspicious data and missing data will cause great influence on on-line PFR performance analysis and assessment of WAMS data, so some compensation measures for WAMS data have to be taken to the suspicious data and missing data.

\section{A. Suspicious Data Processing}

Suspicious data refers to the data that may have some abnormal data qualities or obviously deviate the normal change track of curve in WAMS. Firstly, filter such data with abnormal quality codes as GPS out-of-step, unavailable data and abnormal generator through verification of PMU data quality code. Secondly, filter the active power and frequency of the generator through amplitude limit, for example, when the active power of current generator exceeds 1.5 times of the output limit of the generator or current frequency difference exceeds $0.8 \mathrm{HZ}$, the generator data can be regarded as suspiciousness. Finally, fit the suspicious point with adjacent efficient points or interpolate for auto repair to improve data utilization. PMU measured data through high-density sampling has relatively obvious jitter. If the jump variable of adjacent two frames of generator output sometimes even exceeds $1 \mathrm{MW}$, or even bigger, it needs to obtain the variation trend of measured data by utilizing the average-filtering jitter elimination. Refer to Figure II for the generator data before / after filter. The blue imaginary line represents the generator output after filter.

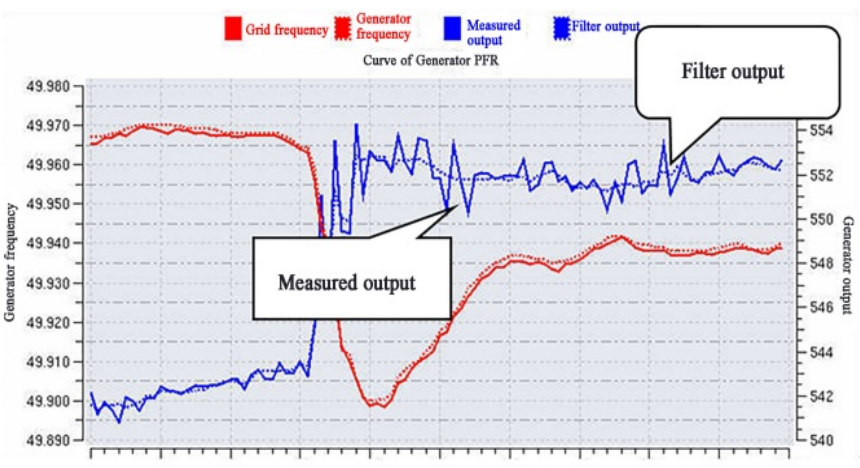

FIGURE II. CURVE OF GENERATOR PFR

\section{B. Missing Data Processing}

Missing data means that the data of individual sampling point is missing or PMU fails to acquire the required data. As individual sampling point loses, the adjacent efficient points can be adopted to fit or such methods as linear interpolation can be adopted for supplement. When PMU fails to acquire the required data, SCADA data can be adopted for supplement in the method of piecewise fitting. Article [7] compares level of similarity and real-time difference of WAMS and SCADA data 
curve. It founds that at same physical measurement point, the lag is less than 1s and the difference of active power value is less than $2 \%$ in the majority through direct acquisition of SCADA data and PMU data. Therefore, current WAMS aligning time scale and SCADA data can be acquired in real time through smart grid dispatching technical supporting system to conduct data time scale aligning, modification and piecewise fitting and supplement the missing data.

\section{COMPREHENSIVE ANALYSIS AND LEAN ASSESSMENT FOR PFR PERFORMANCE}

In this article, the above-mentioned generator PFR performance analysis method principle based on WAMS and SCADA hybrid data source is adopted to dynamically monitor the process of grid frequency variation, capture effective disturbance of the grid frequency, comprehensively analyze characteristic parameters and contribution effects of the generator PFR within different times of frequency fluctuation, and comprehensively analyze the generator PFR performance mainly based on output response index, piecewise energy contribution index, comprehensive index and multiple assessment indexes.

Nevertheless, because the generator PFR performance is affected by operation conditions of generator and other factors, the response performance has larger dynamic characteristics. The characteristic parameter calculated each time may be different from other. Sometimes, they even differ greatly. It cannot be easily regarded the basis of its performance assessment with the data acquired at a certain time. The statistical results of long-term accumulated repeated performance analysis need to be combined for overall assessment. Therefore, this article puts forward lean PFR assessment system on time dimension. This assessment system is divided into two categories: real-time assessment index and long-term statistical index.

\section{A. Real-time Assessment Index}

Real-time assessment index is to assess the generator PFR performance from the angle of single performance. As per the calculation results of single generator frequency regulation performance, obtain the comprehensive assessment index through weighted fusion of relevant performance indexes of regulating speed, regulation range and regulation precision to realize the comprehensive assessment of PFR performance. In addition, according to the section where the index is located, set comment set $V=$ (excellent, good, medium, qualified, failed, reverse regulation) to assess the generator PFR performance. Real-time assessment index mainly includes response lag time, output response index, energy contribution index and comprehensive index.

\section{B. Long-time Statistical Index}

Long-time statistical index is to assess the generator PFR performance from the angle of long-time performance. As per the calculation results of repeated generator PFR performance, conduct long-period statistics to PFR performance, and realize the comprehensive assessment of PFR performance according to the comprehensive assessment index. Statistical index includes average regulating speed, average regulation range, average regulation precision, comprehensive response index, effective disturbing times, assessment times and excellent times.

\section{ON-LINE TESTING OF PFR PERFORMANCE}

The on-line generator PFR performance monitoring function can reflect the PFR performance response of generator under actual operating environment through on-line capturing and real-time analysis, but it cannot conduct PFR performance analysis to the shutdown generator during the frequency disturbance because the PFR assessment lacks of comprehensiveness. On the other hand, it might be disturbed by seriously suspicious data and missing data, especially when the frequency farther deviates from the standard frequency, the PFR regulating variable and automatic gain control (AGC) regulating variable will superpose together to affect the on-line PFR performance analysis results. Therefore, against the above situations, this article puts forward the combination of on-line generator PFR performance testing function to perfect and supplement the analysis and assessment method for generator PFR performance.

The on-line PFR performance testing is based on the appointed testing interactive mechanism at dispatching side and power plant side. The master station issues PFR switch-in, switch-out and various types of test instructions in the way of telecommand issuance. After the receipt of corresponding instructions, the substation simulates the PFR operation conditions of the system under LF or HF operation conditions to realize PFR increase / decrease tests, continuous increase / decrease tests and characteristic parameter test functions. In view of poorer timeliness of remote terminal unit (RTU) data acquisition of the substation, correctly grasp the operating time and operating power of generator PFR through analysis verification of basic data and test results by adopting the test mode of parallel processing of RTU and PMU double data sources, to ensure the accuracy of test results. Refer to Figure III for the generator response curve in on-line PFR performance test. The blue curve is the actual generator output curve.

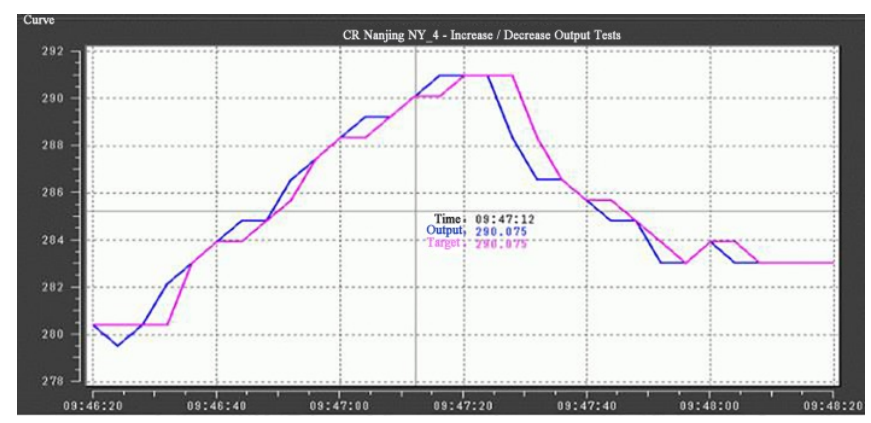

FIGURE III. ON-LINE PFR TEST BASED ON PMU DATA

\section{FIELD APPLICATION}

By utilizing the above principles and methods, based on the smart grid dispatching technical supporting system, NARI Technology Co., Ltd. develops comprehensive analysis and lean assessment software for PFR of generator based on 
WAMS/SCADA hybrid measurements. At present, the software has had actual application in Jiangsu Power Grid.

Specific field application example: At 03:05 on October 20, 2015, East China Power Grid broke down in DC monopole blocking, which caused that the grid frequency dropped to 49.764 HZ within 10 seconds. The on-line PFR performance monitoring and assessment software acquired this frequency disturbance. The PFR performance calculated through analysis was basically in line with the actual operation. Refer to Figure IV for the analysis and assessment results for PFR performance of Da-tang Nanjing \#2 Generator based on PMU data. Refer to Fig. 5 for the analysis and assessment results for PFR performance of Da-tang Nanjing \#2 generator based on RTU data.

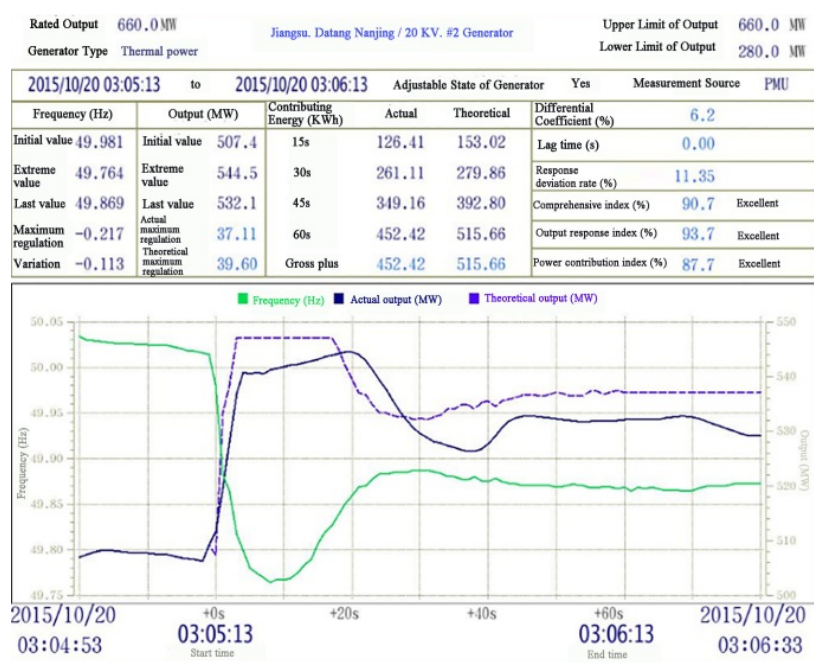

FIGURE IV. ANALYSIS AND ASSESSMENT RESULTS FOR PFR OF GENERATOR BASED ON PMU DATA

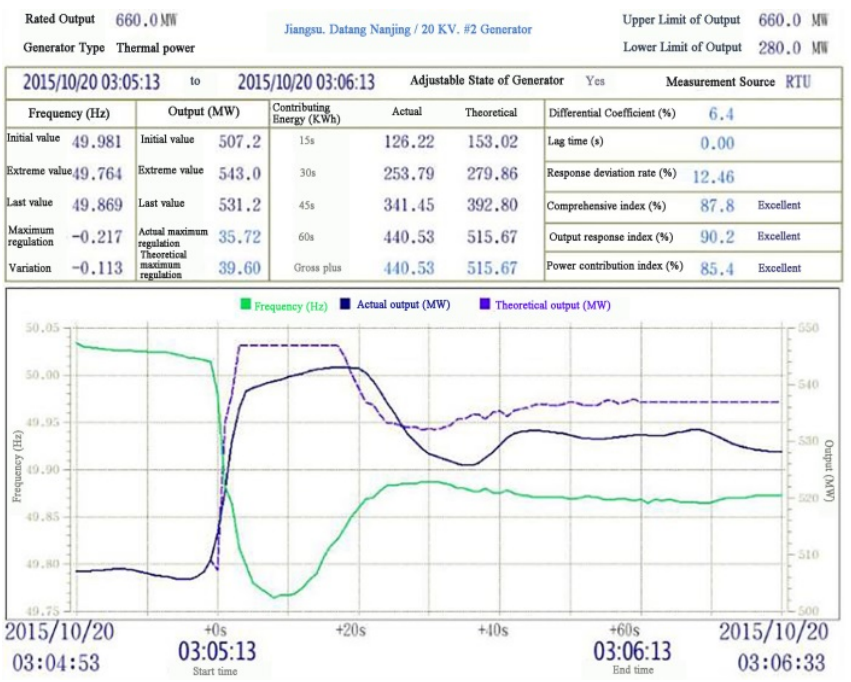

FIGURE V. ANALYSIS AND ASSESSMENT RESULTS FOR PFR OF GENERATOR BASED ON RTU DATA

From Figure IV and Figure V, it can be seen that the fitting degree of actual output curve and theoretical output curve is high in the process of PFR, indicating better generator PFR performance. In Table 1, the regulating speed index, regulation range index, regulation precision index and PFR response index calculated respectively based on WAMS/SCADA are basically the same, and the conditions reflected by the real-time assessment index is basically in line with the actual operation conditions, indicating better single performance for PFR performance of Da-tang Nanjing \#2 Generator. In Table II, the statistical results of long-term indexes of Da-tang Nanjing \#2 Generator in September and October are recorded respectively. From the results of this table, it can be seen that the PFR performance of Da-tang Nanjing \#2 Generator has better longterm performance.

TABLE I. REAL-TIME ASSESSMENT INDEXES FOR PFR OF GENERATOR

\begin{tabular}{|c|c|c|c|c|c|c|}
\hline Generator & $\begin{array}{c}\text { Generat } \\
\text { or } \\
\text { Capacity } \\
\text { MW }\end{array}$ & $\begin{array}{c}\text { Regulat } \\
\text { ing } \\
\text { Speed } \\
\%\end{array}$ & $\begin{array}{c}\text { Regulat } \\
\text { in } \\
\text { Range } \\
\%\end{array}$ & $\begin{array}{c}\text { Regulat } \\
\text { in } \\
\text { Precisio } \\
\mathbf{n} \%\end{array}$ & $\begin{array}{c}\text { Compreh } \\
\text { ensive } \\
\text { Index \% }\end{array}$ & Remarks \\
\hline $\begin{array}{c}\# 2 \\
\text { Generato } \\
\mathrm{r}\end{array}$ & 660 & 82.6 & 93.7 & 88.9 & 90.7 & $\begin{array}{c}\text { PMU } \\
\text { data }\end{array}$ \\
\hline $\begin{array}{c}\# 2 \\
\text { Generato } \\
\mathrm{r}\end{array}$ & 660 & 82.4 & 90.2 & 86.9 & 87.8 & $\begin{array}{c}\text { RTU } \\
\text { data }\end{array}$ \\
\hline
\end{tabular}

TABLE II. LONG-TERM STATISTICAL INDEXES FOR PFR OF GENERATOR

\begin{tabular}{|c|c|c|c|c|c|c|}
\hline Generator & $\begin{array}{c}\text { Generat } \\
\text { or } \\
\text { Capacity } \\
\text { MW }\end{array}$ & $\begin{array}{c}\text { Regula } \\
\text { ting } \\
\text { Speed } \\
\%\end{array}$ & $\begin{array}{c}\text { Regula } \\
\text { ton } \\
\text { Range } \\
\%\end{array}$ & $\begin{array}{c}\text { Regula } \\
\text { tion } \\
\text { Precisi } \\
\text { on \% }\end{array}$ & $\begin{array}{c}\text { Compreh } \\
\text { ensive } \\
\text { Index \% }\end{array}$ & Remarks \\
\hline $\begin{array}{c}\# 2 \\
\text { Generato } \\
\mathrm{r}\end{array}$ & 660 & 83.2 & 93.4 & 79.5 & 86.5 & October \\
\hline $\begin{array}{c}\# 2 \\
\text { Generato } \\
\mathrm{r}\end{array}$ & 660 & 73.1 & 79.5 & 79.7 & 79.6 & $\begin{array}{c}\text { Septemb } \\
\text { er }\end{array}$ \\
\hline
\end{tabular}

Meanwhile, Jiangsu Power Grid conducted on-line tests to the PFR performance of Da-tang Nanjing \#2 Generator based on PMU data after the event. In Table III, the real-time assessment indexes calculated by the on-line PFR testing of Da-tang Nanjing \#2 Generator are recorded. The index assessment results are same to the on-line monitoring assessment results.

TABLE III. TESTING INDEXES FOR PFR OF GENERATOR

\begin{tabular}{|c|c|c|c|c|c|c|}
\hline Generator & $\begin{array}{c}\text { Generat } \\
\text { or } \\
\text { Capacit } \\
\text { y MW }\end{array}$ & $\begin{array}{c}\text { Regula } \\
\text { ting } \\
\text { Speed } \\
\%\end{array}$ & $\begin{array}{c}\text { Regulat } \\
\text { ion } \\
\text { Range } \\
\text { \% }\end{array}$ & $\begin{array}{c}\text { Regulat } \\
\text { ion } \\
\text { Precisio } \\
\text { n \% }\end{array}$ & $\begin{array}{c}\text { Compre } \\
\text { hensive } \\
\text { Index \% }\end{array}$ & Remarks \\
\hline $\begin{array}{c}\# 2 \\
\text { Generator }\end{array}$ & 660 & 86.2 & 95.4 & 90.1 & 92.8 & $\begin{array}{c}\text { On-line } \\
\text { test }\end{array}$ \\
\hline
\end{tabular}

Both real-time assessment index and long-term statistical index comprehensively indicate better PFR performance of Datang Nanjing \#2 Generator, which are same to the previous actual operation conditions and regular test results. Thus it can be seen that the comprehensive analysis and lean assessment 
method for PFR of generator based on WAMS/SCADA hybrid measurements can accurately assess the PFR effect of the generator.

\section{CONCLUSIONS}

This article realizes the comprehensive analysis and assessment of PFR of generator based on WAMS and SCADA hybrid data and wide-area measured information of the generator. This method can objectively and comprehensively analyze and assess the PFR performance of heterogeneous generators within different times of frequency fluctuation. The assessment results are real and reliable. They can accurately reflect the actual dynamic response conditions of PFR system. The performance parameters and assessment indexes obtained can be regarded as the important reference for performance assessment of PFR system.

\section{REFERENCES}

[1] He Yang-zan, Wen Zeng-yin. Electric Power System Analysis. Rev. 3. Wuhan: Huazhong University of Science \& Technology Press. 2002

[2] Dai Yiping, Zhao Ting, Gao Lin. Research on Characteristics of Power System Primary Frequency Control Operating on Power Plans [J]. Electric Power, 2006, 39 (11): 37-41

[3] Luo Jian-yu, Wang Xiao-ying, Lu Ting-rui, et al. An Application of Power System Real-time Dynamic Monitoring System Based on Widearea Measurement. Automation of Electric Power System, 2003, 27 (24): 78-80

[4] Zheng Tao, Gao Fu-ying. On-line Monitoring and Computing of Unit PFR Characteristic Parameter Based on PMU [J]. Automation of Electric Power Systems, 2009, 33 (11): 57-61, 71.

[5] Li Duan-chao, Chen Shi, Chen Zhong-yuan, Lu Jin-dian. Real-time Measurement and Reward Method of the Efficiency of Generator Unit Primary Frequency Regulation [J]. Automation of Electric Power Systems, 2004, 28 (2): 70-72.

[6] Zhang Yan-jun, Gao Kai, Qu Zu-yi. An Evaluation Method of Primary Frequency Modulation Performance Based on Characteristics of Unit Output Power Curves [J]. Automation of Electric Power Systems, 2012, 36 (7): 99-102.

[7] Qi Wen-bin, Xiao Xiao-chun, Li Dong-ping, Xu Xing-wei, Shao Guanghui. The Difference between SCADA and WAMS Real-Time Data in Dispatching Center. Southern Power System Technology, 2013, 7 (5): 87-9. 